\title{
Oral Candidiasis: An Opportunistic Infection of AIDS
}

\section{Mary Ann Jabra-Rizk*}

Associate Professor, Department of Oncology and Diagnostic Sciences, Dental School Department of Microbiology and Immunology, School of Medicine Department of Pathology, School of Medicine University of Maryland, Baltimore, USA

Candida albicans (C. albicans) is an opportunistic fungal species commonly colonizing human mucosal surfaces. However, when host defenses are weakened, $C$. albicans can proliferate causing an array of infections ranging from mucosal to systemic infections that are often life-threatening. Specifically, oral candidiasis is considered an AIDSdefining opportunistic infection with up to $80 \%$ of HIV+ individuals suffering recurrent episodes during the course of their HIV disease progression. As a dimorphic microbial species, C. albicans is capable of switching morphology between yeast and hyphal forms. Where the yeast form is responsible for systemic disease, hyphae are invasive filaments and therefore are adept at causing mucosal infections. The mouse model is the most suitable animal model to study the development of oral candidiasis due to the similarity to the disease process in humans. (A) Following anesthesia, animals are infected sublingually using swabs saturated with C. albicans cell suspensions. (B) Five days post-infection, white lesions are seen on tongue surface rapidly developing into overt candidiasis characterized by white plaques covering the tongue and other oral surfaces. In order to visualize the process of tissue invasion by $C$. albicans, animals are euthanized and the tongues are harvested and subjected to histopathology analysis where tongue tissue are embedded in paraffin, sectioned and stained with Periodic Acid Schiff stain then examined by light microscopy. (C) Microscopic images of tissue sections demonstrate the extensive presence and penetration of epithelial tissue by the invasive $C$. albicans hyphae (white arrows) with massive influx of host immune cells (yellow arrows). Tongues were also subjected to scanning electron microscopy analysis. (D) Magnified (5000x) images of the tongue surface demonstrate penetration of hyphae from the sublingual area where C. albicans is inoculated leaving sizable gaps at sites of emergence of the hyphae through the tongue surface (red arrows). This extensive tissue invasive process by C. albicans hyphal elements is manifested clinically as white lesions characterizing oral candidiasis.

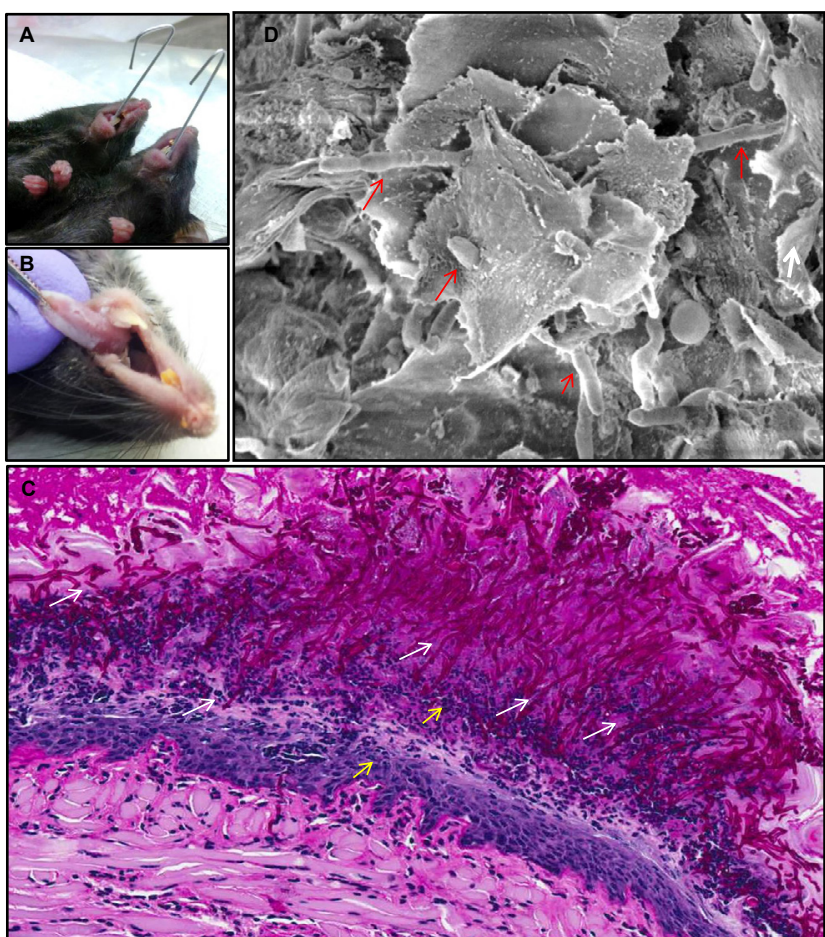

Figure 1
*Corresponding author: Mary Ann Jabra-Rizk, Associate Professor, Department of Oncology and Diagnostic Sciences, Dental School Department of Microbiology and Immunology, School of Medicine Department of Pathology, School of Medicine University of Maryland, Baltimore, USA, Tel: 410-706-0508; E-mail: mrizk@umaryland.edu

Received August 18, 2014; Accepted August 21, 2014; Published August 28, 2014

Citation: Jabra-Rizk MA (2014) Oral Candidiasis: An Opportunistic Infection of AIDS. J AIDS Clin Res 5: i101. doi:10.4172/2155-6113.1000i101

Copyright: @ 2014 Jabra-Rizk MA. This is an open-access article distributed under the terms of the Creative Commons Attribution License, which permits unrestricted use, distribution, and reproduction in any medium, provided the original author and source are credited. 